\title{
Timing behavior in squirrel monkeys as a function of positive vs. negative reinforcement
}

\author{
Evalyn F. Segal and Duane M. Rumbaugh \\ SAN DIEGO STATE COLLEGE
}

\begin{abstract}
Abstraet
Four squirrel monkeys were trained for 88 sessions on a multiple schedule that included two DRL $10 \mathrm{sec}$. components. In one, timing responses were reinforced by the presentation of sucrose pellets; in the other, by the offset of aversive noise for $20 \mathrm{sec}$. (1) Timing was better under positive reinforcement. (2) Noise was an effective negative reinforcer. (3) Timing under positive reinforcement did not improve markedly from about the 25 th to about the 88 th session, but timing under negative reinforcement worsened. (4) The noise-offset contingencies may have opposed the development of accurate timing.

\section{Problem}

The aims of this experiment were (1) to determine whether noise-offset is an effective reinforcer for squirrel monkeys; (2) to compare the effectiveness of food, and noise-offset, as reinforcers of spaced responding (timing); and (3) to compare timing behavior at two stages of training.

\section{Method}

Four young adult male squirrel monkeys were maintained at $80 \%$ of ad lib weight and given daily training sessions of $2-5 \mathrm{hr}$. in ventilated, sound-resistant, Lehigh (model 1417) squirrel monkey conditioning chambers. Monkeys A and C worked always in chamber 1; monkeys $B$ and $D$ in chamber 2. The front wall of each chamber contained two Lehigh rat levers, and immediately above them, two Lehigh pigeon keys, which could be transilluminated by lamps of different colors. Inside the sound-resistant shell, but outside, behind, and to the left of the front wall of the conditioning chamber proper, was a speaker through which white noise could be fed from a sound system that included a Grason-Stadler noise generator (model 455B), a Heathkit amplifier (model W7-A), and a system of relay circuitry that permitted presentation of no noise, continuous noise, or pulsating (once per sec.) noise, A pellet dispenser containing $45 \mathrm{mg}$ Noyes sucrose pellets emptied into a food cup mounted on the front wall, between the two levers. A water spout intruded to the right of the food cup, and water was always available.

The monkeys were shaped to press the right lever for sucrose and the left lever to turn off the noise; this arrangement was constant thereafter. When a $\mathrm{right}$ lever press was appropriate, the key above the right lever was lit, and the other key was dark. When a left lever press was appropriate, the left key was lit. The monkeys were trained in gradual stages, over a month, to the following multiple reinforcement schedule: DRL $10 \mathrm{sec}$. (sucrose; yellow light) S $\Delta$ (both keys, dark) FR 15 (sucrose; green light) S $\triangle \mathrm{DRL} 10 \mathrm{sec}$. (noiseoffset for 20 sec.; red light) $\mathrm{S} \Delta$. Each component of the schedule was 3 min. long, and 5-12 cycles of the complete (18-min.) schedule constituted an experimental session. All monkeys received equal numbers of cycles on a given day.

With the white noise on continuously, the noise level inside the chamber, in the corner nearest the speaker, was $103 \mathrm{db}$ (SPL). With the noise off, it was $82 \mathrm{db}$. With the noise pulsating, it was 94-95 db in chamber 1 , and 95-96 db in chamber 2. During the first month's pretraining we found that continuous noise was a less effective negative reinforcer than pulsating noise; with the noise on continuously, the monkeys remained immobile in a corner of the chamber.
(This may relate to the finding of Biederman et al. (1964) that pulsating shock was more effective than continuous shock in converting an escape response to an avoidance response, in rats.) The final schedule used only pulsating noise as the negative reinforcer.

The first response in each occurrence of a DRL component was always reinforced; thereafter, and for the duration of the component, only responses meeting the $10-\mathrm{sec}$. spacing requirement were reinforced. During the sucrose-reinforced component, this resulted in reinforcing every lever press spaced 10 or more sec. from the preceding right lever press. During the negatively reinforced component, the contingencies were more complex. Each timing interval began only when the preceding 20-sec. no-noise reinforcement interval ended. If no response occurred during the first $10 \mathrm{sec}$. of noise, the next left lever press terminated the noise for $20 \mathrm{sec}$. However, as in the positively-reinforced DRL component, each premature response reset the timing interval, so that a $\mathrm{l}$ le as $\mathrm{s}$ 10 sec. without a lever press, but with noise present, had to elapse before a response cquld terminate the noise for $20 \mathrm{sec}$.

Training under the final schedule parameters continued for 88 daily sessions. During sessions $21-25$ and $85-88$, print-out data were collected and later converted to inter-response-time (IRT) and inter-reinforcement-time (IS $\mathrm{R}_{\mathrm{T}}$ ) distributions. Data were recorded from one of the two chambers on odd-numbered cycles, and from the other chamber on even-numbered cycles, assignment of "odd" and "even" to the two chambers varying unsystematically from day to day. Equal numbers of cycles contributed to the distributions for each monkey.

\section{Results}

Figure 1 shows each monkey's IRT and ${ }^{2} \mathrm{R}_{\mathrm{T}}$ distributions for sessions 21-25, summed; Fig. 2 shows the comparable data for sessions 85-88. Shaded areas in the IRT distributions represent reinforced IRT's. The numerals near each distribution indicate the number of observations contributing to the distributions. Note that far fewer data contributed to the noise-off than to the sucrose distributions. This was partly a limitation

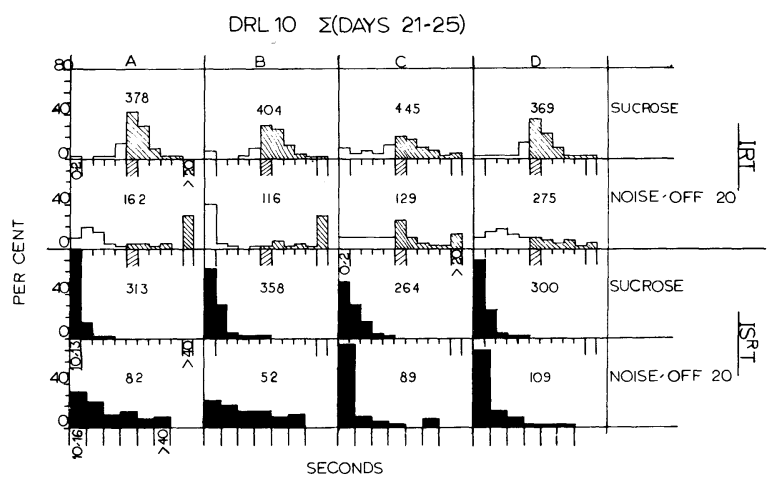

Fig. 1. Summed IRT and IS $^{\mathrm{R}} \mathrm{T}$ distributions for each $\mathrm{S}$, sessions 21-25. Class intervals for IRT distributions are 0-2 sec., 2-4 sec., etc.; for sucrose-reinforced ${ }{ }^{R} \mathrm{~T}$ distributions, 10-13 sec., 13-16 sec., etc; for noise-offset IS $^{\mathrm{R}_{\mathrm{T}}}$ distributions, 10-16 sec., 16-22 sec., etc. 


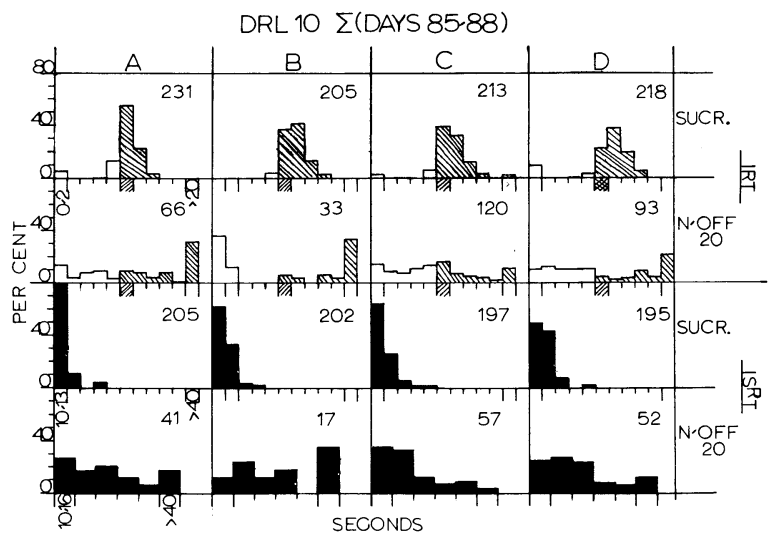

Fig. 2. Summed IRT and $\mathrm{IS}^{\mathrm{R}} \mathrm{T}$ distributions for sessions $85-88$.

imposed by the procedure: every reinforcement during a noise component subtracted $20 \mathrm{sec}$. from the total of $3 \mathrm{~min}$. available for making responses and obtaining reinforcements. Partly, it was a result of the poor response spacing that occurred on the noise-offset schedule. Note also that five days of observations contributed to the distributions of Fig. 1, but only four days to Fig. 2.

The notable features of the distributions are: (1) Each monkey showed a characteristic set of distributions, different from the other monkeys, and more or less stable from earlier to later in training. (2) Each monkey's food-reinforced distributions were markedly stable from earlier to later training; i. e., the additional training did not appreciably improve response spacing. (3) Positively reinforced timing was good, as shown by the height of the IRT distributions at 10-12 sec., by the rarity of premature responses in general, and of "burst" $(0-2$ sec. IRT) responses in particular, and by the height of the IS $\mathrm{R}_{\mathrm{T}}$ distributions in the first (10-13 sec.) interval. (4) Negatively reinforced timing was so poor that inspection of the IRT distributions shows little evidence of temporal discrimination. However, the IS $^{R_{T}}$ distributions reveal that at least two of the monkeys, $\mathrm{C}$ and $\mathrm{D}$ (one from each of the chambers), were timing, although not very accurately. (5) From earlier to later in training, negatively reinforced timing worsened, as revealed by the flattening of the ${ }_{1 S}{ }^{R_{T}}$ distributions.

In the face of the poor timing on the noise-offset sechedule, it is important to note that noise-offset wa s reinforcing left lever presses. By sessions 21-25, and thereafter, each monkey made only $0-2$ left lever presses during non-noise portions of a cycle; i. e., almost all left lever presses occurred during the component in which such responses were reinforced by noise offset.

\section{Diseussion}

The worsening of timing on the noise-offset schedule, from the earlier to the later sessions, may indicate a progressive long-term adaptation to the noise, so that it may have become less aversive, and its offset therefore less reinforcing. There may have been damage to the hearing apparatus, although the monkeys appeared normal to casual observation.

The characteristics of the noise-offset schedule itself may have worked against the development of accurate response spacing. First, the monkeys were required to tolerate at least $10 \mathrm{sec}$. of aversive noise before a response could terminate the noise for $20 \mathrm{sec}$. Exposure to a minimum of $10 \mathrm{sec}$. of noise per timing interval may have (a) elicited emotional responses incompatible with, or disturbing to, timing behavior; (b) produced shortterm (as well as long-term) adaptation to the noise, so that it became less aversive by the end of $10 \mathrm{sec}$. (although pulsing the noise should have minimized shortterm adaptation); or (c) favored the conditioning of other noise-reducing operants at the expense of lever pressing; e. g., sitting facing into the corner farthest from the speaker, hands over ears. Second, the monkeys were required to approach the noise in order to terminate it: the speaker was located behind, and slightly to the left, of the left lever, so that approaching the left lever brought the monkey as close as possible to the noise source. This may have had a punishing effect on left lever pressing. Third, each timing interval began anew 20 sec. after the last successful timing response. This amounts to a variant of a discrete-trials DRL procedure (Logan, 1961; Zimmerman, 1961). If successful response spacing depends on the conditioning of collateral response chains, then imposing a 20-sec. delay between one timing interval and the next might oppose the development of stable forms of response chaining, and thereby interfere with accurate timing.

As results indicate only slight success in the development of timing behavior with noise as a negative reinforcer, we conclude that the present procedures were probably not optimal.

\section{References}

BIEDERMAN, G. B., D'AMATO, M. R., \& KELLER, D. M. Facilitation of discriminated avoidance learning by dissociation of $\mathrm{CS}$ and manipulandum. Psychon. Sci., 1964, 1, 229-230.

LOGAN, F. A. Discrete-trials DRL. J.exp. Anal.Beh., 1961, 4, 277-279.

ZIMMERMAN, J. Spaced responding in rats as a function of some temporal variables. J. exp. Anal. Beh., 1961, 4, 219-224.

\section{Note}

1. Supported by NASA contract 14413, NSF grant GB 1605, and NIMH grant 08505 . 